\title{
Discovery of Eight Recycled Pulsars - The Swinburne Intermediate Latitude Pulsar Survey
}

\author{
Russell T. Edwards \\ Centre for Astrophysics 85 Supercomputing, Swinburne University of \\ Technology Mail 31 PO Box 218 Hawthorn VIC 3122 A ustralia
}

\begin{abstract}
We have conducted a pulsar survey of intermediate Galactic latitudes $\left(5^{\circ}<|b|<15^{\circ}\right)$ at $20 \mathrm{~cm}$. The survey has been highly successful, discovering 58 new pulsars, eight of which are recycled, in only $\sim 14$ days of integration time. One pulsar has a very narrow ( $2^{\circ} \mathrm{FWHM}$ ) average profile for the pulsar's period $(278 \mathrm{~ms})$. The six new recycled binary systems provide valuable information on the formation of white dwarf pulsar binaries. Two systems have massive white dwarf companions (> $0.57 \mathrm{M}_{\odot}$ and $\left.>1.2 \mathrm{M}_{\odot}\right)$, while another has a low mass $\left(\sim 0.2 \mathrm{M}_{\odot}\right)$ companion in a $23.3-\mathrm{d}$ orbit, residing the well-known orbital period "gap".
\end{abstract}

\section{The Swinburne Intermediate Latitude Pulsar Survey}

Full details of the observing hardware and analysis procedures are available in Edwards et al. (2000). Briefly, 265-s pointed observations were made with the 64-m Parkes radiotelescope using the sensitive new $21 \mathrm{~cm} 13$-beam receiver. The backend system includes twenty-six filterbanks, each with ninety-six channels and a total bandwidth of $288 \mathrm{MHz}$, centred at a sky frequency of 1374 $\mathrm{MHz}$. Detected filterbank outputs are summed in polarisation pairs and one-bit digitised with an integration time of $125 \mu \mathrm{s}$. Data is recorded on magnetic tape for offline processing on the Swinburne supercluster, a network of 64 Compaq Alpha workstations, using standard techniques (e.g. Manchester et al. 1996).

The survey area has been observed and processed to a completeness of $90 \%$ and has been highly successful with a minimal investment of telescope time, discovering 58 new pulsars to date. Of these, eight are recycled, six of which are in binary systems with circular orbits, indicating white dwarf companions.

\section{Discovery Highlights}

We have discovered a pulsar with a period of $278 \mathrm{~ms}$ and an average pulse profile only $W \simeq 2$. 1 FWHM. Rankin (1990) observed that the distribution of pulsar profile widths for core-type pulsars is well fit by the constraint $W P^{1 / 2}>2.45$, where $P$ is the pulsar period in seconds. The newly discovered pulsar J1410$7407^{1}$, however, has $W P^{1 / 2}=1$. 1 . Preliminary polarimetric results at $660 \mathrm{MHz}$

\footnotetext{
${ }^{1}$ Pulsar name is subject to change due to the present uncertainty in its position
} 
and $1400 \mathrm{MHz}$ indicate that the profile has two components, furthering the discrepancy between the observed component widths of this pulsar and other long-period systems.

The orbital period distribution of low mass binary pulsars previously appeared to include a "gap" (Camilo 1994) in the range $12.4<P_{\text {orb }}(\mathrm{d})<56.3$. A number of authors (e.g. Kulkarni 1995, Tauris 1996) have suggested that this gap separates those systems that evolved with significant angular momentum losses from those that did not. Systems with an orbital period less than the socalled "bifurcation period", $P_{\text {bif }}=1-2$ days, undergo orbital contraction during mass transfer due these losses. The newly discovered binary system J1618-39191 has an orbital period of 23.3 days, placing it in the middle of the "gap". Further to the considerations above, we suggest that there is a narrow range of initial orbital periods (significantly longer than $P_{\text {bif }}$ ) over which (for increasingly close orbits) angular momentum losses quickly become significant. This results in an under-density of systems with final orbits in the range of $7-60$ days, particularly around the upper end of this range (the former "gap"). The distribution around $P_{\text {bif }}$ appears fairly even.

The mass functions of two of the new recycled binary systems indicate that the companion is a massive $\mathrm{CO}$ or $\mathrm{ONeMg}$ white dwarf - for J1757-5322 $M_{\mathrm{WD}}>0.57 \mathrm{M}_{\odot}$, whilst for $\mathrm{J} 1157-5112 M_{\mathrm{WD}}>1.2 \mathrm{M}_{\odot}$. Five recycled binary pulsars with massive white dwarfs were previously known (see e.g. Arzoumanian, Cordes \& Wasserman 1999). It has been suggested that the evolution of these systems included a deep common envelope phase (van den Heuvel 1994). PSR $\mathrm{J} 1757-5322$ is in a very close 11-hour orbit (with an orbital separation of $a \simeq 3.1$ $R_{\odot}$ ) and may be explained by this model only if an energy source other than orbital decay largely powers envelope ejection. J1157-5112, on the other hand, is only marginally compatible with the model of van den Heuvel (1994) due to the relatively wide 3.5 -day orbit $\left(a \simeq 13.5 \mathrm{R}_{\odot}\right)$.

Acknowledgments. I thank the Galactic plane multibeam pulsar survey collaboration (see Camilo et al. 1999) for their assistance of our use of equipment built for that survey and helpful discussions, and Swinburne collaborators $M$ Bailes, M Britton and $W$ van Straten.

\section{References}

Arzoumanian, Z., Cordes, J.M., \& Wasserman, I. 1999, ApJ, 520, 696.

Camilo, F. 1994 in "The Lives of Neutron Stars". eds Alpar, A., Kiziloglu U., van Paradijs, J., Kluwer, Dordrecht. 243

Camilo, F. et al. 1999, this volume

Edwards, R.T. et al. 2000, ApJ, in prep.

Kulkarni, S.R. 1995, in "Millisecond Pulsars: A Decade of Surprise". eds Fruchter, A.S., Tavani, M. \& Backer, D.C., ASP. 79.

Manchester, R.N. et al. 1996, MNRAS, 279, 1235

Rankin, J. 1990, ApJ, 352, 247

Tauris, T.M 1996, A\&A, 315, 453.

van den Heuvel, E.P.J. 1994, A\&A, 291, L39 